\title{
Theory of self phase-locked optical parametric oscillators
}

\author{
J.-J. Zondy and A. Douillet \\ Laboratoire Primaire du Temps et des Fréquences \\ Bureau National de Métrologie / Observatoire de Paris \\ 61, avenue de l'Observatoire, F-75014 Paris, France. \\ A. Tallet, E. Ressayre and M. Le Berre \\ Laboratoire de Photophysique Moléculaire \\ Batiment 210, Université de Paris Sud, 91405, Orsay Cedex, France.
}

(October 26, 2018)

\begin{abstract}
The plane-wave dynamics of $3 \omega \rightarrow 2 \omega, \omega$ subharmonic optical parametric oscillators containing a second harmonic generator of the idler-wave $\omega$ is analyzed analytically by using the meanfield approximation and numerically by taking into account the field propagation inside the media. The resonant $\chi^{(2)}(-3 \omega ; 2 \omega, \omega): \chi^{(2)}(-2 \omega ; \omega, \omega)$ cascaded second-order nonlinearities induce a mutual injection-locking of the signal and idler waves that leads to coherent self phase-locking of the pump and subharmonic waves, freezing the phase diffusion noise. In case of signal-and-idler resonant devices, largely detuned sub-threshold states occur due to a subcritical bifurcation, broadening out the self-locking frequency range to a few cavity linewidths.
\end{abstract}

PACS: 42.65.-k, 42.65.Yj, 42.65.Sf, 42.65.Ky

\section{INTRODUCTION}

The synthesis of phase-coherent (sub)harmonic optical radiation $(\omega, 2 \omega, \ldots, N \omega)$ is useful in high precision optical measurements, such as optical frequency metrology [1], [2]. In frequency metrology, a phase-coherent optical by- $N$ divider allows to reduce the absolute measurement of an optical frequency $N \omega$ (e.g, couting cycles in the hundreds of $\mathrm{THz}$ range) to the measurement of a smaller frequency interval $\Delta=\omega$ by use of a femtosecond laser comb generator whose radio-frequency intermode spacing is phase-locked to a primary microwave clock [3]. The generation of a comb of phase-locked harmonic radiation can also be the starting basis for the generation of attosecond pulse train by Fourier synthesis [4], [5], [6]. Indeed, the superposition (Fourier synthesis [7], [8]) of a comb of $N$ equal amplitude optical harmonic fields with a controllable relative phase relationship can lead to a temporal train of ultra-short pulses with a sub-optical cycle duration $(\tau \sim 2 \pi / N \omega)$ and a repetition rate equal to the fundamental harmonic $\omega$. Phase-locked optical harmonic generators are often based on many lasers linked via nonlinear upconversion parametric processes [1], [9], [10], 11], 12]. Phase coherence among the harmonic waves is usually achieved by use of complex electronic phase-locking loops. The high conversion efficiencies of optical parametric oscillators (OPOs) combined with the engineering flexibility offered by periodically poled (PP) nonlinear materials may allow a compact implementation of such optical subharmonic generators or attosecond pulse generator.

The study of OPOs as by- $N$ dividers $(N=2,3,4)$ of a pump frequency $\nu_{p}$ is motivated by their capacity to perform the phase-coherent division of a pump photon into two highly phase-correlated subharmonic photons. In precision measurement setups, subharmonic generation leads to a subsequent phase noise reduction $\left(\div N^{2}\right)$ compared to harmonic generation $\left(\times N^{2}\right)$. Graham and Haken have first demonstrated that the phase sum $\varphi_{1}+\varphi_{2}$ of the idler and signal waves from an OPO follows adiabatically the phase noise $\varphi_{p}$ of the pump laser, while their phase difference $\varphi_{2}-\varphi_{1}$ undergoes a phase diffusion process stemming from the interaction of both modes with the vacuum fluctuations [13. To act as pertinent phase-coherent dividers, the idler and signal waves have thus to be phase-locked, for instance by use of an electronic servo control that forces $\varphi_{2}-\varphi_{1}$ to copy the phase of an external RF oscillator referenced to a primary clock. Such an electronic servo technique has been implemented to develop phase-coherent divide-by-2 (2:1) [14], and divide-by-3 (3:1) OPOs for high resolution optical metrology [15], [16] with corresponding residual phase difference variance well below $1 \mathrm{rad}^{2}$.

Electronic phase-locking loops are however subject to limited response time bandwidth and require fast OPO cavity length actuators. As an alternative, all-optical self phase-locking (SPL) methods are currently being investigated [17], 
[18] to by-pass the bandwidth limitation of electronic servos and simplify the implementation of phase-locked dividers. These methods are based on the self injection-locking of the subharmonic waves, similarly to the injection locking process of a slave laser oscillator by a master laser which possesses a better spectral purity and frequency stability [7], [19]. In injection-locked systems, the phase of the slave oscillator, $\phi(t)=\omega t+\varphi(t)$, is a perfect copy of the phase of the injecting master oscillator. Phase locking can occur only when the two frequencies are close enough, e.g. within a certain locking range $\Delta \omega_{\text {lock }}=\left|\omega_{s}-\omega_{m}\right|$ whose extent is proportional to the squared ratio $\sqrt{P_{m} / P_{s}}$ of the injecting master power to the slave power. It is thus expected that the self-locking range in an OPO divider should depend on whether the OPO is configured as a doubly-resonant (DRO), a triply-resonant (TRO) or a pump-enhanced singly resonant oscillator (PRSRO). Strong self injection-locking regime occurs only when the signal and idler waves are simultaneously resonant.

For divide-by-2 OPOs based on a type-I nonlinear process (the signal and idler are identically polarized), selfinjection locking occurs naturally when the OPO is tuned close enough to the frequency-degeneracy [20]. Recently a type-II self phase-locked 2:1 DRO was demonstrated, in which a small mixing of the two orthogonally polarized signal and idler waves, performed by an intracavity wave plate, induces the injection locking [17]. In both cases the evidence of self phase locking was provided by the high level of phase-coherence between the frequency degenerate signal and idler waves. Following the experiment in ref [17], the theory of linearly coupled type-II 2:1 SPL-DROs has been reported 21]. In the case of 3:1 OPO dividers, obviously such a linear coupling cannot be implemented, and one must use a nonlinear coupling via $\chi^{(2)}(-3 \omega ; 2 \omega, \omega): \chi^{(2)}(-2 \omega ; \omega, \omega)$ cascading processes (for instance by introducing inside the OPO cavity a second nonlinear material phase-matched for the SHG of the idler wave). The first implementation of such a self phase-locked (SPL) OPO was recently reported, and used a dual-grating periodically poled lithium niobate chip carrying the OPO and SHG sections in a pump-resonant idler-resonant configuration (PRSRO) 18]. An extremely small locking range of $\sim 500 \mathrm{kHz}$, corresponding to a fraction of the idler cavity linewidth, was reported due to the weak (non resonant) doubled idler power. A different situation should occur when both the signal and idler waves are resonant, as in a DRO or a TRO. Because of the enhanced energy flow exchange between the $2 \omega$ and $\omega$ modes, the dynamics of such a self-phase locked OPO (SPL-OPO) is expected to differ significantly from that of a conventional OPO. Strictly speaking the 3:1 SPL-DRO or TRO can be regarded as a degenerate sub/second harmonic generator $(2 \omega \leftrightarrows \omega+\omega)$ internally pumped by the signal wave of a non-degenerate OPO $(3 \omega \leftrightarrows 2 \omega+\omega)$. While theories of OPO devices containing additional up or down conversion of the signal and idler waves have been investigated in the past with the aim of generating new frequencies [22], [23], [24], [25], or revealing the quantum noise signature of competing $\chi^{(2)}$ nonlinearities [26], [27] such a subharmonically resonant configuration of competing nonlinearities in OPOs has never been theoretically investigated in detail. A 3:1 DRO-SHG rough rate equation analysis based on photon flux conservation, but neglecting the role of cavity detunings and field phase coherence, was previously given by Zhang et al [28] with the conclusion that self-phase locking should manifest through an imbalance of the signal and idler intensities at exact 3:1 division. Actually our in-depth analysis shows that such an imbalance holds only for zero detunings: When the nonlinear phase shift due to cascading is compensated by a non-zero optimal cavity detuning, one can obtain the same output intensities as in a conventional DRO.

In this paper, we provide a detailed plane-wave description of the dynamics of divide-by-3 SPL-OPOs that encompasses all the three cavity configurations (only the main results are summarized in the conclusion for the PRSRO, which will be detailed elsewhere). The dynamics of the nonlinearly coupled OPO is shown to differ substancially from the linear coupling case of a type-II 2:1 SPL-DRO [21]. In particular, the nonlinear coupling gives rise to a subcrital bifurcation for any non-zero cavity detuning while conventional OPOs undergo subcritical bifurcations only for the case of largely pump-detuned TROs [29], 30]. As a consequence of the nonlinear phase shifts due to the cascading processes, SPL-DROs or TROs will be shown to display large-intensity stable stationary states that would correspond to non-lasing detuning domains in conventional OPOs, extending thus the phase-locking detuning range up to a few cavity linewidths.

This paper is organized as follows. In section II we describe the basic plane-wave ring cavity model leading to the meanfield solutions that will be compared with the full propagation solutions. In section III, we first treat in detail the DRO configuration case. We then extend the theory to the case of a TRO in section IV. Main results on the PRSRO configurations will be also summarized in the conclusion. Section V discusses the practical implementation of such SPL-OPOs on the basis of the theoretical findings and section VI concludes with some prospective studies aroused by this first theoretical approach. 


\section{BASIC MODEL AND EXACT NUMERICAL SOLUTIONS}

The OPO-SHG device described in the paper is schematically sketched in Fig.1. We consider a ring cavity containing the cascaded OPO and SHG nonlinear media, each of length $L_{1}$ and $L_{2}$ respectively and assume that the pump, signal and idler waves satisfy already the 3:2:1 frequency ratios. Though the drawing depicts the case of a dual-grating PP material, phase-matched for an (eee) interaction, the model is valid also for any separate birefringent material sections, or a single material phase-matched for both interactions. In the case of birefringent phase-matching however, the OPO should be of type-II kind (e.g. $e \rightarrow o+e$ or $o \rightarrow e+o$ ) and the SHG of type-I kind (resp. $e+e \rightarrow o$ or $o+o \rightarrow e$ ), so that the system is described by only 3 field variables.

\section{A. Plane-wave propagation equations}

We denote by $z=Z / L_{1}$ and $z^{\prime}=Z / L_{2}$ the normalized propagation distances within each crystal, such that $0 \leq z \leq 1$ between points " 0 " and " 1 " and $0 \leq z^{\prime} \leq 1$ between " 1 " and " 2 ". Let $E_{j}$ (in m/V) be the slowly varying complex field amplitudes where the subscripts $j=p, 2,1$ stand respectively for pump, signal and idler

$$
\epsilon_{j}(Z, t)=\frac{1}{2} E_{j}(Z, t) \exp \left[i\left(\omega_{j} t-k_{j} Z\right)\right]+c . c .
$$

and let $N_{j}$ be the complex field variable, such that $\left|N_{j}\right|^{2}$ is the number of photons in mode $j$ at plane $z$ inside the ring cavity, by

$$
N_{j}=\sqrt{\frac{\epsilon_{0} c V}{2 \hbar \omega_{j}}} E_{j}
$$

where $V$ is the average volume occupied by the modes inside the resonator. Then, the reduced field amplitudes $A_{j}$ are introduced by scaling $N_{j}$ with the small signal gain coefficient $g_{1} L_{1}$ of the OPO crystal

$$
A_{j}=g_{1} L_{1} N_{j}
$$

with

$$
g_{1}=\frac{d_{O P O}}{c} \sqrt{\frac{2 \hbar \omega_{1} \omega_{2} \omega_{p}}{\epsilon_{0} V n_{1} n_{2} n_{p}}}
$$

In Eq.(4), $d_{O P O}=\mathbf{e}_{p} \cdot\left[\chi^{(2)}(-3 \omega ; 2 \omega, \omega) / 2\right]: \mathbf{e}_{2} \cdot \mathbf{e}_{1}$ (in $\left.\mathrm{m} / \mathrm{V}\right)$ is the effective nonlinear coefficient for the OPO interaction and $n_{j}$ are the refractive indices at frequency $\omega_{j}$. The reduced Maxwell equations for these field amplitudes $A_{j}(\mathrm{z}, \mathrm{t})$, when propagating through the $(3 \omega \rightarrow 2 \omega, \omega)$ crystal without any diffraction effect, are

$$
\begin{aligned}
& \frac{d A_{p}}{d z}=i A_{1} A_{2} \\
& \frac{d A_{2}}{d z}=i A_{p} A_{1}^{*} \\
& \frac{d A_{1}}{d z}=i A_{p} A_{2}^{*}
\end{aligned}
$$

using the standard slowly varying amplidude approximation and the usual change of variables $z \longrightarrow z, t \longrightarrow t-\bar{n} z / c$, where $\bar{n}$ is the mean linear refractive index. In Eqs. 5 perfect phase-matching has been assumed, $\Delta k_{O P O}=k_{p}-$ $k_{2}-k_{1}=0$. The propagation equations in the SHG crystal, allowing for a non-vanishing wavevector mismatch $\Delta k_{S H G}=k_{2}-2 k_{1}$, are

$$
\begin{aligned}
& \frac{d A_{p}}{d z^{\prime}}=0 \\
& \frac{d A_{2}}{d z^{\prime}}=i S A_{1}^{2} \exp \left(+i 2 \xi z^{\prime}\right) \\
& \frac{d A_{1}}{d z^{\prime}}=i S A_{2} A_{1}^{*} \exp \left(-i 2 \xi z^{\prime}\right)
\end{aligned}
$$


with initial conditions $A_{j}\left(z^{\prime}=0\right)=A_{j}(z=1)$.

The two cascaded crystals are usually phase-matched by adjusting their temperature or angle. But, exact zero mismatch for both interactions may be difficult to achieve, so that a phase-mismatch parameter $\xi=\Delta k_{S H G} L_{2} / 2$ is introduced. The parameter $S$ in eqs. (6) is the ratio of the SHG to OPO small signal gains

$$
S=\frac{g_{2} L_{2}}{g_{1} L_{1}}
$$

where the small signal gain SHG coefficient $\left(\right.$ in $\left.^{-1}\right)$ is

$$
g_{2}=\frac{d_{S H G}}{c} \sqrt{\frac{2 \hbar \omega_{1}^{2} \omega_{2}}{\epsilon_{0} V n_{1}^{2} n_{2}}}
$$

For a type-I (eee) PP crystal the effective nonlinear coefficients satisfy $d_{O P O} \approx d_{S H G}$, so that with $n_{1} \approx n_{2} \approx n_{p}$ and $\omega_{p}=3 \omega, \omega_{2}=2 \omega, \omega_{1}=\omega$, the relation $(7)$ can be approximated by $S \approx\left(L_{2} / L_{1}\right) / \sqrt{3}$. For $L_{2} / L_{1}=1 / 3$ for instance, one has $S \approx 0.2$.

The solutions at the SHG crystal exit can be limited to few terms of the Mac-Laurin field expansion serie at the cell entrance, because of the smallness of the parametric gain coefficients. We shall refer to ML1 approximation, keeping only the lowest-order perturbative terms, i.e. quadratic terms in powers of the field amplitudes. At the exit of the SHG crystal, the ML1 axpproximation provides the solutions for the signal and idler amplitudes as functions of their value at the entrance of the OPO crystal (point "0" in Fig.1)

$$
\begin{aligned}
& A_{p}\left(t, L_{1}+L_{2}\right)=A_{p}(t, 0)+i A_{1}(t, 0) A_{2}(t, 0) \\
& A_{2}\left(t, L_{1}+L_{2}\right)=A_{2}(t, 0)+i A_{p}(t, 0) A_{1}^{*}(t, 0)+i \chi^{*} A_{1}^{2}(t, 0) \\
& A_{1}\left(t, L_{1}+L_{2}\right)=A_{1}(t, 0)+i A_{p}(t, 0) A_{2}^{*}(t, 0)+i \chi A_{2}(t, 0) A_{1}^{*}(t, 0)
\end{aligned}
$$

with the nonlinear coupling constant $\chi$

$$
\chi=S \exp (-i \xi)(\sin \xi / \xi)
$$

\section{B. Boundary conditions}

The reduced Maxwell equations have to be completed by the boundary conditions for the three waves at the entrance of the OPO crystal to derive the cavity equations. The field $A_{j}(t+\tau, 0)$ at location " 0 " and at a time $t+\tau$, where $\tau=\Lambda / c$ is the cavity roundtrip time ( $\Lambda$ is the total cavity optical path), is the field $A_{j}\left(t, L_{1}+L_{2}\right)$ at point "2" of Fig.1, which propagates freely after bounces at the totally reflecting $(R=1)$ and output coupling $\left(r_{j}, t_{j}\right)$ mirrors and eventually summed with an input field, as it is the case for the pump field. We define by $r_{j}\left(t_{j}\right)$ the overall mirror amplitude reflectivities (transmissivities), such that $r_{j}^{2}+t_{j}^{2}=1$ and define the amplitude loss coefficients $\kappa_{j}=1-r_{j}$.

Then, the boundary conditions take then the form

$$
\begin{aligned}
& A_{p}(t+\tau, 0)=r_{p} \exp \left(i \Delta_{p}\right) A_{p}\left(t, L_{1}+L_{2}\right)+A_{i n} \\
& A_{2}(t+\tau, 0)=r_{2} \exp \left(i \Delta_{2}\right) A_{2}\left(t, L_{1}+L_{2}\right) \\
& A_{1}(t+\tau, 0)=r_{1} \exp \left(i \Delta_{1}\right) A_{1}\left(t, L_{1}+L_{2}\right)
\end{aligned}
$$

where $A_{i n}$ denotes the input pump amplitude inside the cavity, $A_{\text {in }}=t_{p} A_{i n}^{\text {ext }}$. Each $\Delta_{j}$ is the usual cavity detuning between the frequency of the waves and the corresponding cold cavity frequency, scaled to the HWHM cavity resonance width ( Only nearly resonant waves will be considered, $\Delta_{j}<<2 \pi$ ).

The set of equations (9) and (11) forms a mapping of the field amplitudes at location "0", from which meanfield equations may be derived.

The meanfield model was originally derived from a similar set of equations for a two-level cell ring cavity device when the atomic dephasing time is much greater than the round-trip time $\tau$, but much smaller than the photon lifetime $\tau / \kappa$ [31. For an OPO, the situation is somewhat different because the response of the crystal is assumed to be instantaneous (see Eqs. 5). Nevertheless, meanfield equations can be also derived from the ML1 equations (9) and the boundary conditions (11) if the field amplitudes are slowly varying during a round-trip time, i.e. $\tau\left(d A_{j} / d t\right)<<A_{j}(t)$ [32], [33]. 
In the conventional TRO case $\left(|\chi|=0, \Delta_{j}<<1, \kappa_{j}<<1\right)$, nontrivial homogeneous stationary solutions exist for the idler and the signal only if the following relation holds [29], [30]

$$
\bar{\Delta}=\Delta_{1} / \kappa_{1}=\Delta_{2} / \kappa_{2} .
$$

and the intracavity pump intensity $I_{p}=\left|A_{p}\right|^{2}$ is clamped to the threshold input intensity,

$$
I_{t h}=\kappa_{1} \kappa_{2}+\Delta_{1} \Delta_{2}
$$

whatever the input intensity $\left|A_{\text {in }}\right|^{2}$ might be above the threshold. In the case of the DRO, the relation (12a) still holds. But solutions for the signal and idler intensities can be determined only if the expansion (9) is continued up to the cubic terms, leading to

$$
\begin{aligned}
I_{1} & =2 \kappa_{2}\left[\sqrt{1+\left(1+\bar{\Delta}^{2}\right)\left(I_{p} / I_{t h}-1\right)}-1\right] \\
I_{1} / I_{2} & =\Delta_{2} / \Delta_{1}=\kappa_{2} / \kappa_{1} \\
\cot \left(\varphi_{p}-\varphi_{1}-\varphi_{2}\right) & =-\bar{\Delta}
\end{aligned}
$$

The bifurcation is supercritical for any detuning . Besides, the phase difference $\varphi_{1}-\varphi_{2}$ is an undetermined quantity while the sum phase $\varphi_{1}+\varphi_{2}$ depends on the pump laser phase [21]. Actually, this classical phase indetermination is compatible with the result of the quantum fluctuation theory of parametric oscillators [13].

\section{SPL-DRO CASE}

\section{A. Stationary solutions}

In the case of a DRO, only the signal and idler waves resonate with the cavity while the pump wave is a travelling wave $\left(t_{p}=1, r_{p}=0\right)$. Assuming $\kappa_{1,2} \ll 1, \Delta_{1,2} \ll 1$, Eqs.(9-11) provide the stationary solutions, $A_{j}(t+\tau, 0)=$ $A_{j}(t, 0) \equiv A_{j}$,which are also those of the meanfield model,

$$
\begin{aligned}
A_{p} & =A_{i n} \\
\left(\kappa_{2}-i \Delta_{2}\right) A_{2} & =i A_{p} A_{1}^{*}+i \chi^{*} A_{1}^{2} \\
\left(\kappa_{1}-i \Delta_{1}\right) A_{1} & =i A_{p} A_{2}^{*}+i \chi A_{2} A_{1}^{*}
\end{aligned}
$$

Eqs (13) can be conveniently solved by setting $A_{j}=\alpha_{j} \exp \left(i \varphi_{j}\right)$, giving rise to

$$
\begin{aligned}
\left(\kappa_{2}-i \Delta_{2}\right) \alpha_{2} & =i \alpha_{p} \alpha_{1} \exp (i \mu)+i|\chi| \alpha_{1}^{2} \exp (-i \eta) \\
\left(\kappa_{1}-i \Delta_{1}\right) \alpha_{1} & =i \alpha_{p} \alpha_{2} \exp (i \mu)+i|\chi| \alpha_{1} \alpha_{2} \exp (+i \eta) \\
\mu & =\varphi_{p}-\varphi_{2}-\varphi_{1} \\
\eta & =\varphi_{2}-2 \varphi_{1}-\xi
\end{aligned}
$$

The non-trivial solutions $\left(A_{j} \neq 0\right)$ can be easily handled by setting $X=\left(|\chi| \alpha_{1}\right)^{2}$ and $I_{p}=\alpha_{p}^{2}$. Then the scaled idler intensity is found to be solution of

$$
X^{2}-2\left(I_{p}-\kappa_{1} \kappa_{2}+\Delta_{1} \Delta_{2}\right) X+\left|\left(\kappa_{1}-i \Delta_{1}\right)\left(\kappa_{2}+i \Delta_{2}\right)-I_{p}\right|^{2}=0
$$

Let us note that the coupling terms in Eqs.(14) allow to obtain the non-trivial solutions at the ML1 approximation order, unlike for the conventional DRO.

Three cases have to be distinguished in order to solve Eq. (15), $\Delta_{1,2}=0, \Delta_{1} \Delta_{2} \neq 0$ (but with $\Delta_{1} \Delta_{2}>0$ ), and $\Delta_{1,2}=0, \Delta_{2,1} \neq 0$.

For $\Delta_{1} \Delta_{2} \neq 0$, there are two solutions

$$
X_{ \pm}=\left(|\chi| \alpha_{1}\right)_{ \pm}^{2}=I_{p}-\kappa_{1} \kappa_{2}+\Delta_{1} \Delta_{2} \pm 2 \sqrt{\Delta_{1} \Delta_{2}\left[I_{p}-I_{0}\right]}
$$

for any input $I_{p} \geq I_{0}$, where $I_{0}$ is the input intensity at the saddle-node bifurcation 


$$
I_{0}=\frac{1}{4}\left(\kappa_{1} \sqrt{\frac{\Delta_{2}}{\Delta_{1}}}+\kappa_{2} \sqrt{\frac{\Delta_{1}}{\Delta_{2}}}\right)^{2}
$$

while the threshold intensity, determined by $d X_{-} / d I_{p}=0$, is

$$
I_{t h}=I_{0}+\Delta_{1} \Delta_{2}
$$

which is minimum for $\Delta_{1} / \kappa_{1}=\Delta_{2} / \kappa_{2}$ and equal to $\kappa_{1} \kappa_{2}+\Delta_{1} \Delta_{2}$, like for the conventional DRO.

The signal intensity $I_{2}$ is related to $I_{1}$ via the relation

$$
\left(\alpha_{1} / \alpha_{2}\right)^{2}=\Delta_{2} / \Delta_{1}
$$

deduced from Eqs.(14a-b), so that the two detunings must have the same sign.

The solutions $X_{+}$and $X_{-}$are represented by the lines a and a', respectively, in Fig. 2 that displays a subcritical bifurcation: Indeed, only the stationary solution $X_{+}$is stable ( solid line a ) and extends from the saddle-node intensity $I_{0}$ to the Hopf bifurcation intensity $I_{H}$, above which the solution is periodic (dashed portion). The stationary solution $X_{-}$is marginally unstable (dashed line a'), whatever the input intensity might be [See Appendix].

Let us notice that the conventional DRO detuning condition, $\bar{\Delta}=\Delta_{1} / \kappa_{1}=\Delta_{2} / \kappa_{2}$ does no longer necessarily hold and hence the SPL-DRO can oscillate with a wider detuning range. This result is similar to the result of a $2: 1$ degenerate SPL-DRO, in the case of a linear coupling. However, this latter case does not display any subcriticality: The linear coupling gives rise instead to two self phase-locked states corresponding to two distinct thresholds, for a given pumping rate [21].

The phases $\mu$ and $\eta$ fulfill the relations

$$
\begin{aligned}
\sin \mu & =-\frac{1}{2 \alpha_{p}}\left[\kappa_{1} \sqrt{\frac{\Delta_{2}}{\Delta_{1}}}+\kappa_{2} \sqrt{\frac{\Delta_{1}}{\Delta_{2}}}\right]=-\sqrt{\frac{I_{0}}{I_{p}}} \\
\sin \eta & =-\frac{1}{2|\chi| \alpha_{1}}\left[\kappa_{1} \sqrt{\frac{\Delta_{2}}{\Delta_{1}}}-\kappa_{2} \sqrt{\frac{\Delta_{1}}{\Delta_{2}}}\right] \\
\text { with } \alpha_{p} \cos \mu+|\chi| \alpha_{1} \cos \eta & =-\sqrt{\Delta_{1} \Delta_{2}}
\end{aligned}
$$

Eqs.(20) completely determine the absolute phases of the subharmonic waves, which are hence self-locked. These relations on the idler and signal phases, while similar to those in ref [21], will be shown to present substantial differences. As a consequence of the self-locking, highly phase-coherent subharmonic outputs are expected from the SPL-DRO.

In the case $\Delta_{1,2}=0$, the bifurcation is supercritical with

$$
\begin{aligned}
X & =I_{p}-I_{t h} \\
I_{t h} & =\kappa_{1} \kappa_{2}
\end{aligned}
$$

The real and imaginary parts of Eqs. (14a-b) give rise to

$$
\begin{aligned}
\sin \mu & =-\frac{1}{2 \alpha_{p}}\left[\kappa_{1} \frac{\alpha_{1}}{\alpha_{2}}+\kappa_{2} \frac{\alpha_{2}}{\alpha_{1}}\right] \\
\sin \eta & =-\frac{1}{2 \sqrt{I_{p}-\kappa_{1} \kappa_{2}}}\left[\kappa_{1} \frac{\alpha_{1}}{\alpha_{2}}-\kappa_{2} \frac{\alpha_{2}}{\alpha_{1}}\right] \\
\alpha_{p} \cos \mu+\sqrt{I_{p}-\kappa_{1} \kappa_{2}} \cos \eta & =0
\end{aligned}
$$

Eq.(22c) implies that $\cos \mu$ and $\cos \eta$ are either of opposite signs or simultaneously nil. The first case corresponds to

$$
\frac{\alpha_{1}}{\alpha_{2}}=\frac{\kappa_{1}}{\kappa_{2}}=1 \text { with } \sin \eta=0 \text { and } \sin \mu=-\frac{\kappa_{1,2}}{\alpha_{p}}
$$

The case $\cos \mu=\cos \eta=0$ is compatible only with $\kappa_{1} \neq \kappa_{2}$, leading to 


$$
\begin{aligned}
\frac{\alpha_{1}}{\alpha_{2}} & =\sqrt{\frac{\kappa_{2}}{\kappa_{1}}}(\sqrt{N} \mp \sqrt{N-1}) \\
\sin \eta & = \pm 1 \text { and } \sin \mu=-1
\end{aligned}
$$

where $N=I_{p} / \kappa_{1} \kappa_{2}$ is the pumping rate. Eq.(24a) displays a dependence of $\frac{\alpha_{1}}{\alpha_{2}}$ on the pump amplitude, unlike eq.(19) and consequently the solutions are not continuous when $\Delta_{1,2} \rightarrow 0$, in the general case $\kappa_{1} \neq \kappa_{2}$.

For $\Delta_{1,2}=0, \Delta_{2,1} \neq 0$, Eq. (15) has no real solution. Actually the exact solutions will be shown to display a time-dependent regime.

Finally let us note that the signal and idler intensities are inversely proportional to $|\chi|^{2}$ as displayed by Eq.(16). This dependence is a consequence of the ML1 approximation, Eqs.(9). (The limit $|\chi| \rightarrow 0$ is irrelevant since the meanfield equations (13) are misleading for the conventional DRO).

\section{B. Numerical results}

The time evolution of the field amplitudes is obtained by solving the propagation equations (5-6) with the boundary conditions (11) for a given input amplitude and small initial signal and idler amplitudes. The solutions are obtained by numerical integration of Eqs. (5-6) using of a fourth-order Runge Kutta algorithm, until convergence is achieved. In most of the calculations, the cavity loss coefficients are taken constant and equal, $\kappa_{1,2}=0.005$, and the SHG phase mismatch $\xi=\Delta k L_{2} / 2=0$, unless otherwise stated. The input amplitude, the detunings and the coupling constant are varied.

\section{- Stationary solutions}

As predicted by the linear stability analysis of the meanfield solutions, a single solution, corresponding to $X_{+}$, occurs by numerical integration for an input amplitude above the threshold given by Eq.(18), where the trivial solution is unstable. In a general manner, the signal and idler intensities satisfy the relations predicted by the mean-field, either for non-zero detunings in Eq. (19) or vanishing detunings in Eqs. (23-24). Nevertheless, for zero detunings, numerical intensities agree with the meanfield intensities, only for small coupling parameters $(|\chi| \leqslant 0.15)$. As already pointed out, there is no continuity for the intensities when $\Delta_{1,2} \rightarrow 0$ in the case $\kappa_{1} \neq \kappa_{2}$.

The exact stationary amplitude $|\chi| \alpha_{1}$ and its stability domain depend on the magnitude of $|\chi|$ as displayed in Fig. 2 by line $b$ for $|\chi|=0.05$ and line $c$ for $|\chi|=0.2$. In this latter case, the exact solution is close to the meanfield one, the stationary solution extends almost from $I_{0}$ in the subthreshold domain, reachable only by backward adiabatic decrementation of the pump intensity, to the Hopf bifurcation threshold intensity $I_{H}^{\text {num }}$, slightly larger than the meanfield value. As $|\chi|$ decreases, the domain of stability is shortened below threshold $\left(I_{0}^{\text {num }}>I_{0}\right)$, but it is enlarged above threshold, because the Hopf bifurcation threshold is shifted towards larger input intensity, $I_{H}^{\text {num }}>I_{H}$ ( For $\kappa_{1,2}=\Delta_{1,2}=0.005$, the meanfield predicts $I_{H}=2 I_{t h}$, while the numerical values are $I_{H}^{\text {num }} \simeq 2.6 I_{t h}$ for $|\chi|=0.2$ and $I_{H}^{\text {num }}>4 I_{\text {th }}$ for $\left.|\chi|=0.05\right)$.

Subcriticality is also evidenced in Fig.3, when starting the numerical integration from $\bar{\Delta}=0$ and performing an adiabatic increase of $\bar{\Delta}$, for a fixed input pump intensity $I_{p}=4 \kappa_{1} \kappa_{2}$. The different curves in solid line correspond to different values of $|\chi|$. They are symmetric for negative detunings, so that the tuning curve of the SPL-DRO appears as a widened double-sided fringe, which reminds of resonantly phase-modulated Fabry Perot devices (the same kind of fringe is obtained from a resonator containing an electro-optic phase modulator driven by a RF oscillator whose frequency is equal to the resonator free spectral range [34] $)$. In the case of the conventional DRO $(|\chi|=0$, dashed

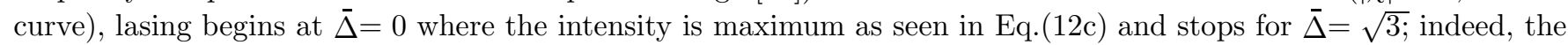
bifurcation is supercritical so that lasing may occur only if the input pump intensity is larger than the threshold value $\kappa_{1} \kappa_{2}+\Delta_{1} \Delta_{2}$. The case $|\chi|=0.01$ (curve a), corresponding to nascent bistability, displays lasing, approximately in the same range of detuning as in the $|\chi|=0$ case, with the significant difference that there is self phase-locking (see below on Fig.6). As $|\chi|$ increases further, the bifurcation becomes subcritical, the saddle-node moves away from the threshold, approaching the meanfield location, independently of $\bar{\Delta}$. The intensity reaches the same maximum $I_{\max }$ for any $0 \leq|\chi| \leq 0.25$, but at a detuning $\bar{\Delta}_{\max }(|\chi|)$, for which the pump is entirely depleted; then the intensity decreases to zero when increasing further the detuning. The maximum $I_{\max }$ is equal to the maximum conventional DRO intensity, which occurs at $\bar{\Delta}_{\max }=0$. In case of the parameters used in Fig.3, Eq. (12c) gives rise to $\sqrt{I_{\max }}=0.1$, in agreement with the numerical result. The detuning $\bar{\Delta}_{\text {max }}$ becomes approximately proportional to $|\chi|$ for large enough coupling strength. For $|\chi| \geq 0.25$, the idler intensity becomes time-dependent before $\bar{\Delta}_{\max }$ is reached.

In summary, the cascaded SHG nonlinearity induces a $|\chi|$ - dependent phase that shifts the optimum detuning from 0 to $\bar{\Delta}_{\max }$. 
The temporal response of the system subject to stepwise-like detuning jumps has been also studied in order to check the stability of the subthreshold states against external perturbations that tend to modulate the detuning parameter (via the cavity length for instance). Figure 4, associated with $|\chi|=0.2$, shows that the system recovers its steady state operating point as long as the perturbation amplitude does not exceed $\pm 0.12 \bar{\Delta}_{\text {max }}$. Note the longer decay time, which is characteristic of a critical slowing-down phenomenon, on the positive detuning step side that brings the DRO very close to the saddle-node bifurcation.

We can define a self-locking domain in the $\left(\Delta_{1}, \Delta_{2}\right)$ plane over which the DRO is self phase-locked with a well-defined phase relationship. Contour plots of the numerical solutions for the idler $I_{1}$, the signal $I_{2}$, the total subharmonic intensity $I_{1}+I_{2}$ and the phase difference $\eta$ are shown in Figs $\mathbf{5}$ for $|\chi|=0.2$ and for a given pumping rate $N=$ $I_{p} / \kappa_{1} \kappa_{2}=4$. The subharmonic intensities are scaled to the conventional zero-detuning DRO values as deduced from Eqs.(12c), $I_{1,2}^{D R O}=2 \kappa_{2,1}$. These contour plots are obtained by adiabatical following of the stationary solutions in order to reveal the $2 \mathrm{D}$ subthreshold domain. The granular small regions adjacent to the $\Delta_{1}=0$ axis correspond to a time-dependent regime. The $I_{1}$ and $I_{2}$ intensity distributions in Figs 5a-5b display off-diagonal maxima that are larger than the conventional DRO signal and idler outputs $\left(I_{1,2} / I_{1,2}^{D R O}=1.6\right)$. Note also that the exact intensities $I_{1}$ and $I_{2}$ are not invariant with respect to the product $\Delta_{1} \Delta_{2}$, differently from the predicted meanfield intensities (16) but they satisfy the relation (19). The figure (5c) shows that the maximum total intensity, slightly larger $\left(I_{1}+I_{2}=2.2\right)$ than the conventional DRO total intensity, $I_{1}+I_{2}=2$, occurs in the subthreshold domain close to the saddle-node bifurcation (see diagonal line d in Fig.3). These curves show that the relative phase control of the subharmonics can be achieved via the control of the relative output intensities, provided that an independent control of both cavity detunings is implemented (see section V).

\section{- Phase-locking}

The numerical phase $\eta$ related to the difference between the signal and idler phases agrees extremely well with the mean-field prediction in Eqs. (20b),( 22b) or (23),(24b) for any values of the detuning and cavity loss parameter, but the numerical phase $\mu$ related to the sum of the signal and idler phases is found to depart significantly from the meanfield value (20a) when $\Delta_{1,2} / \kappa_{1,2} \succsim 1$. We have checked numerically the self phase-locking effect of the signal-idler output, by varing randomly the initial phase of the signal and idler noise. This general result is illustrated in Fig.6, for $\Delta_{1} / \kappa_{1}=\Delta_{2} / \kappa_{2}$ where the phase difference $\eta$ follows randomly the initial values (blank circles), when $|\chi|=0$, as expected for a conventional DRO. However, yet for a vanishingly small value $S=0.001$, the phase difference $\eta$ locks to 0 or $\pi(\bmod k \pi)$ for $\xi=0$ and the sum phase locks to a constant value determined only by the pumping rate.

\section{- Time-dependent solutions}

For $\Delta_{1,2} \neq 0$ and $\Delta_{1} / \kappa_{1}=\Delta_{2} / \kappa_{2}$, the periodic solution remains generally stable on a large range of the input intensity. For instance, for $|\chi|=0.2$ and $\bar{\Delta}=1$ (case of line $\mathrm{c}$ in Fig.2), the periodic solution is stable until the pump parameter reaches $\alpha_{p} / \alpha_{t h} \sim 12$, well above any experimentally achievable input. The period of the amplitude oscillation in the vicinity of $I_{H}^{\text {num }}$ is found to be $T \sim 300 \tau$. But the case $\left|\Delta_{1} / \kappa_{1}-\Delta_{2} / \kappa_{2}\right| \neq 0$ leads to a more complex dynamics, which depends on the nonlinear coupling $|\chi|$ and departs from the meanfield predictions .

In the zero-detuning case, no time-dependent solutions are found for realistic pumping rate $(N<25)$ for equal cavity loss $\kappa_{1}=\kappa_{2}$. Differently, the case $\kappa_{1} \neq \kappa_{2}$ displays time-dependent regimes, for $|\chi| \succsim 0.2$. In the case $\kappa_{1} / \kappa_{2}=2.5,|\chi|=0.2$ shown in Fig.7, a periodic regime arises at $I_{\text {in }} \cong 2 I_{t h}$ and a weak chaotic pulsating behaviour is found at $I_{i n} \cong 4 I_{t h}$, with a pulsating signal intensity. Vanishingly small detunings $\left(\Delta_{1,2}\right)$ however give rise to steady state solutions satisfying relations (16).

The numerical solutions associated with $\Delta_{1,2}=0, \Delta_{2,1} \neq 0$, are not stationary, as predicted by the meanfield model; they are periodic with time for any input above threshold.

\section{- Influence of SHG phase-mismatch}

We have studied the influence of a moderate SHG phase mismatch $(\xi \neq 0)$ on the dynamical behaviour of the system. This phase mismatch is usually controlled by the temperature or the angular orientation of the SHG crystal. It provides a control of the strength of the nonlinear coupling parameter $|\chi|$, via the relation (10), and offsets the value to which the phase difference $\eta$ locks (Fig.6). For instance, a small phase $\xi \leq 1$ does not significantly change the idler amplitudes of Fig.3 but induces a slight imbalance of the signal and idler intensity ratio compared to the ratio (19) and slightly reduces the self-locking range. The relation $\mu=0(\bmod \pi)$ is still valid and $\eta$ locks to $\xi$. In some undesirable operating conditions, for instance, when the system approaches a Hopf bifurcation for a given pumping rate and detuning set (see Fig.2 for $S=0.2, \xi=0$ ), a small amount of phase mismatch produces a shift of the periodic oscillation threshold towards higher pump rate. Differently, for $\bar{\Delta}=0$ and the same other parameters as for curve (c) 
of Fig.2, a small phase mismatch $(\xi=0.1)$ leads to a slow periodic regime $\left(T \sim 4 \times 10^{+6} \tau\right.$ at $\left.\alpha_{i n} / \alpha_{t h}=1.2\right)$ which is suppressed when vanishingly small detunings (always present in practical devices) are introduced.

From the simulations carried out, we conclude that a moderate SHG phase-mismatch does not change significantly the main bifurcation dynamics studied up to this point, except for the $\Delta_{1,2}=0$ case.

Finally let us point out that the SPL-DRO solutions expanded up to the second-order in Eq. (9) (ML2 approximation) agree very well with the exact solutions in almost the whole range of the pump intensity and detunings below the Hopf bifurcation threshold, except for the singular case $\Delta_{1,2}=0$ corresponding to the transition from subcriticality to supercriticality, where it fails to converge. Only the propagation model can solve this case, which requires a double-precision computation due to the slow convergence associated to a critical slowing-down phenomenon.

\section{SPL-TRO CASE}

In the case of SPL-TRO, the meanfield equations (13b)-(13c) are still valid, except that $I_{p}=\alpha_{p}^{2}$ denotes now the circulating intracavity pump intensity. The intracavity pump amplitude obeys the boundary condition (11a), leading to

$$
\left[1-r_{p} \exp \left(i \Delta_{p}\right)\right] A_{p}-i r_{p} \exp \left(i \Delta_{p}\right) A_{1} A_{2}=A_{\text {in }}
$$

where the pump detuning may be arbitrarily large $\left(\Delta_{p} \leq 2 \pi\right)$. The relation between the input pump parameter $I_{i n}=\left|A_{i n}\right|^{2}=\alpha_{i n}^{2}$, and the cavity pump intensity is obtained, for an arbitrary phase of the input field

$$
I_{\text {in }}=\left(1+r_{p}^{2}-2 r_{p} \cos \Delta_{p}\right) \alpha_{p}^{2}+r_{p}^{2} \alpha_{1}^{2} \alpha_{2}^{2}+2 r_{p} \alpha_{1} \alpha_{2} \alpha_{p}\left[\sin \left(\Delta_{p}-\mu\right)+r_{p} \sin \mu\right]
$$

where $\alpha_{1}, \alpha_{2}$ and $\mu$ can be deduced from Eqs.(16) and (19-20). The threshold for oscillation is easily found from Eq. (26), on the basis of simple considerations. Below the threshold, where $\alpha_{1,2}=0, I_{p}$ grows linearly as a function of $I_{\text {in }}$. When it reaches the value given by Eq.(21), oscillation starts, which leads to the following threshold input intensity

$$
I_{t h}^{S P L-T R O}=\left(1+r_{p}^{2}-2 r_{p} \cos \Delta_{p}\right)\left(I_{0}+\Delta_{1} \Delta_{2}\right)
$$

In a practical TROs, although the pump finesse is lower than the finesse at the subharmonic waves (typically an order of magnitude), the condition $\kappa_{p} \ll 1$ is still satisfied. If we consider a small enough pump detuning and the specific case $\bar{\Delta}=\Delta_{1} / \kappa_{1}=\Delta_{2} / \kappa_{2}$, Eq.(27) reduces to the conventional TRO threshold [30]

$$
I_{t h}^{T R O}=\left|A_{i n}\right|_{t h}^{2}=\kappa_{p}^{2} \kappa_{1} \kappa_{2}\left(1+\bar{\Delta}^{2}\right)\left(1+\bar{\Delta}_{p}^{2}\right)
$$

where $\bar{\Delta}_{p}=\Delta_{p} / \kappa_{p}$. Fig. 8 shows the normalized intra-cavity pump and idler bifurcation diagrams as a function of $\sqrt{\bar{I}_{i n}}=\sqrt{I_{i n} / I_{t h}^{T R O}}$ when condition (12a) holds and for $\Delta_{p}=0$. Only the details of the $|\chi| \alpha_{1}$ solutions in the vicinity of the threshold are plotted in the top frame of Fig. 8 that displays the subcriticality. The numerical and meanfield solutions of the idler amplitude as given by Eq. (16) are confounded over a much larger input intensity range. The thick solid and dashed lines for $\alpha_{p}$ correspond to the meanfield solution (26), while the thin solid line is the exact numerical pump solution. Notice that the numerical solution agrees very well with the meanfield solution. As the input parameter is increased from threshold, $I_{p}$ decreases from the clamped value of the conventional TRO to a minimum $I_{0}$ (Eq.17) for an input pump intensity $\bar{I}_{\text {in }}^{\text {min }}$ given by

$$
\bar{I}_{i n}^{\min }=\left[1+\left(\kappa_{1} / \kappa_{p}\right)(\bar{\Delta} /|\chi|)^{2}\right]^{2} /\left(1+\bar{\Delta}^{2}\right)
$$

for $\Delta_{p}=0$. The meanfield model hence predicts that, unlike for conventional TRO, the intracavity pump intensity is not clamped. For $\bar{\Delta} \rightarrow 0, \bar{I}_{i n}^{\min } \rightarrow 1$, e.g. there is a transition to supercriticality as in the SPL-DRO case.

In pump-detuned conventional TROs, subcriticality occurs only when the condition $\bar{\Delta}_{p} \bar{\Delta}>1$ holds [29], [30]. The experimental observation of this subcriticality requires a high pumping level because of the pump detuning dependence in Eq.(28) [35] . It is interesting to study how the subcriticality originating from the nonlinear OPO/SHG cascading would affect the intrinsic detuned TRO bistability curve. In Fig.9 we have plotted the numerically computed idler intensity versus the normalized input intensity for $|\chi|=0,0.2,0.6$ and $\bar{\Delta}_{p} \bar{\Delta}=2$. The subthreshold domain extends 
as $|\chi|$ increases from zero, it has also increased, when compared to the $\bar{\Delta}_{p}=0,|\chi|=0.2$ case, shown in Fig. 8 . Furthermore, as $|\chi|$ increases, the numerical threshold is smaller than the meanfield threshold given by Eq.(27).

We have also verified that the numerical amplitudes and phases agree with the meanfield predictions for $\bar{I}_{\text {in }}<10$ and for detuning values such that $\Delta_{j} / \kappa_{j} \precsim 1$. Far above threshold and for larger detunings, only the phase difference relations remain exact, like in the SPL-DRO case.

Furthermore, the detuning range $\bar{\Delta}$ for self-locking is less dependent on the coupling strength than in the SPL-DRO case.

The high-pump-finesse SPL-TRO device does not exhibit the time-dependent periodical solutions observed above threshold with the SPL-DRO as long as $|\chi| \leq 0.52$ (which corresponds roughly to equal OPO and SHG crystal length for a PPLN) and for realistic maximum pumping rate $\bar{I}_{i n}=I_{i n} / I_{t h}^{S P L-T R O} \leq 2500$, corresponding to a Watt-level pump power and typical TRO pump thresholds in the $\mathrm{mW}$ range. For larger coupling $|\chi|=0.6$, periodic oscillations occur, with a period varying in a very complex way when the input pump is increased. Nevertheless no chaotic regime is observed in the range of considered input pump.

In many practical experiments, one cannot avoid a weak pump resonance in DROs due to the multiwavelength coatings of the mirrors. It is thus interesting to investigate how a moderate pump resonance would affect the $\left(\Delta_{1}, \Delta_{2}\right)$ self-locking domain of Figs.5. The input external pump intensity is kept equal to $I_{i n}^{\text {ext }}=4 \kappa_{1} \kappa_{2}$ to provide a comparison with Figs.5 $(|\chi|=0.2)$. Such a pumping level corresponds to an internal TRO pumping rate $\bar{I}_{i n} / I_{\text {th }}^{T R O}=8 / \kappa_{p}=160$. In Fig. 10, the pump reflectivity is $r_{p}=0.8$ with $\Delta_{p}=0$. Apart from the slight enlargement of the self-locking domains, as compared to Fig.5, which is mainly attributed to the high pumping rate, the intensities are smaller than for the DRO, also the intensity distributions are strongly modified, with off-diagonal maxima.

In summary, the only improvement due to a moderate pump resonance is the stabilization effect respective to the onset of temporal dynamics. The extended self-phase locking range is paid back with lower output intensities.

\section{PRACTICAL IMPLEMENTATION OF SPL-OPOS}

In order to avoid spurious cavity loss, the use of a dual-grating quasi-phase matched periodically poled crystal is particularly well suited to the implementation of SPL-OPOs. However, the grating period should be accurately designed so as to phase-matched simultaneously both interactions for the same chip temperature, even though the theoretical analysis predicts a minor influence of SHG phase mismatch.

From an experimental point of view, it is desirable to implement 3:1 SPL-OPOs using the least constraining OPO configuration. Diagnosis methods to check the high phase coherence between the subharmonics under SPL have to be implemented. In the frequency domain, this can be achieved by monitoring the beatnote between the signal wave and the externally frequency-doubled idler wave (or between the summed subharmonics and the pump). Because under SPL operation these two waves are frequency degenerate, the output signal wave must be preliminary frequencyshifted by a suitable RF frequency $\omega_{R F}$ using, e.g., an acousto-optic modulator. When the OPO operates within the locking range, the beatnote frequency should be fixed to $\omega_{R F}$ and its power spectral density should approach a Dirac function. Another equivalent method to check the phase coherence is to perform an interferometric fringe pattern measurement by overlapping the two beams on a slow detector [17]. In the following, other indirect methods, based on the theoretical analysis will be outlined.

As a starting point of the analysis, we have assumed perfect 3:2:1 frequency ratios for the pump, signal and idler waves. In practical devices, such a situation will be unlikely met at once. The major difficulty will come from the fine (continuous) tuning of the signal and idler frequencies close enough to the 2:1 degeneracy, in order to fall within the capture range of the self-phase locking. For a fixed pump frequency, such a fine tuning is usually performed via the temperature or angle tuning of the phase-matching. Singly resonant devices (PRSROs) offer an easy and relaxed mode-hop free frequency tuning because only one subhamonic wave is resonant, especially in a dual-arm cavity configuration to control independently the pump and signal resonances [36]. However from our analysis of the SPL-PRSRO with a resonant signal wave, which also displays subcriticality, extremely small self-locking ranges are predicted due to the weak coupling between the injecting frequency-doubled idler and the resonant signal. While in a conventional PRSRO the signal field is constrained to oscillate with a nil cavity detuning, the nonlinear coupling is found to allow oscillation over a small detuning range not exceeding the cavity linewidth. The increase of the locking range versus the coupling parameter (e.g. the SHG crystal length) is only moderate, even with $|\chi|=1$ (which would correspond to a SHG crystal $\sim 1.5$ times longer than the OPO crystal supposing that both OPO and SHG interactions have the same nonlinearity magnitude). Due to this limited capture range, experimentally confirmed in ref [18], 3:1 SPL-PRSROs would probably require an additional electronic servo on the cavity length to operate as a stable divider device. A convenient criterion for the assessment of SPL in PRSROs would be the slight enhancement 
of the signal intensity when the nonlinear coupling is switched on, compared to the slight decrease predicted for the SPL-DRO (Fig.3 ). A possible way to realize such a switching is to have an OPO-only grating section patterned beside the OPO/SHG dual-grating section on the periodically-poled wafer.

From our theoretical study, the widest SPL range (a few cavity linewidths) is obtained with the doubly resonant configuration (SPL-DRO) due to the strong self-injection regime, with eventually a weak pump resonance (SPL-TRO) to stabilize the device. Even though the amount of nonlinear coupling required can be extremely small $(|\chi|=0.01)$ - and such low level doubled idler can even be spontaneously generated via non-phase matched or higher-order quasiphase matching in PP single-grating nonlinear OPO crystals - a coupling strength corresponding to $|\chi|=0.1-0.2$ will ensure a robust self-phase locking of the subharmonic wave. Preliminary single mode-pair operation of DRO/TROs usually require an intensity sidelock servo to control the stability of the cavity length. This sidelock servo compares the output signal (or idler) intensity to a stable electronic voltage reference which sets the operating (usually non-zero) signal and idler cavity detuning values. When a linear cavity is used, the signal and idler (plus eventually the pump) detunings cannot be independently controlled via the cavity length. It is then probable that the oscillating mode pair will have equal normalized detunings (condition (12a)) that satisfy the minimum threshold. Under sidelock servo the transition from conventional to SPL states is accompanied necessarily by a detuning transition (see Fig.3). It is then important to set the sidelock reference voltage as close as to the maximum fringe intensity in order that the new detuning value does not exceed the allowed subthreshold range. In the case of a well resolved DRO mode pair cluster, the observation of these subthreshold states, and the associated broadened mode pair fringe, should be made possible via adiabatic cavity length tuning. Such an observation would be an indirect diagnosis of SPL. But it is necessary to have an independent control of the signal and idler detunings to explore the full allowed range of $\left(\Delta_{1}, \Delta_{2}\right)$ detunings depicted in Figs.5 and 10. A dual-cavity DRO/TRO design would then be appropriate. The control of these detunings allows the control of the relative phase between the pump, signal and idler. The output subharmonic intensity ratio $I_{1} / I_{2}$ (see Eq.19) can be used as an error signal for the relative phase control.

\section{CONCLUSIONS AND OUTLOOK}

We have theoretically demonstrated that resonant $\chi^{(2)}: \chi^{(2)}$ nonlinear cascaded OPO/SHG processes induce a self injection-locking between the subharmonic waves of an OPO leading to the self-phase locking of the three interacting waves, unlike in a conventional OPO for which the absolute phases of the signal and idler are undetermined. The theoretical treatment encompasses the meanfield model as well as a full propagation model. The doubly and triply resonant oscillator configurations lead to the widest self phase-locking ranges. The main conclusions are: a) the minimum threshold of oscillation of these devices are identical to the conventional devices; b) the nonlinear cascading leads to a subcritical behaviour, even in the case of a DRO or a PR-SRO, and can lead to the occurrence of self-pulsing instabilitities. This subcriticality is different from the standard subcriticality reported in pump detuned conventional TROs. The nonlinear coupling removes the detuning constraints of conventional systems, allowing for a potentially accurate control of the relative phase between the subharmonic waves. The range of allowable detunings over which the field phases are locked depends on the magnitude of the nonlinear coupling and on the self-injection regime. While this range is smaller than the cavity linewidth for PRSROs (weak injection regime), it spans over several cavity linewidths under strong injection-locking regime obtained in signal/idler resonant devices (DRO/TROs). The SPL-DRO/TRO give rise to a richer dynamics than the singly resonant PRSRO for which no occurence of a Hopf bifurcation is found in the whole range of the system parameters. It is thus interesting to extend the theoretical model by including diffraction effects in order to investigate the possible occurrence of new spatio-temporal dynamics, [33], 37. These self-phase locked OPOs will be useful tools for applications requiring a high degree of optical phase coherence between optical harmonic waves, such as precision optical measurements in the mid-IR or Fourier synthesis of ultra-short optical pulses.

The model developed can be easily extended to the study of divide-by- 4 SPL-OPOs based on the cascading OPO/OPO processes $4 \omega \rightleftarrows 2 \omega \rightleftarrows \omega$ which has the potential to generate up to 8 phase-locked harmonic waves by additional up-conversion processes. Such a strong nonlinearly coupled system can be viewed as a secondary degenerate OPO (DOPO) embedded in a primary DOPO. Our future work will be directed to the theoretical investigation of the stability of 4:1 OPO dividers. A classical signature of the system, derived from the meanfield analysis, is the clamping of the secondary pump $2 \omega$ to the threshold power for the fundamental oscillation.

The present study of 3:1 OPO dividers, which makes the simplest assumption of exact 3:2:1 frequency ratios, arouses another interrogation. An interesting situation not considered regards the behaviour of the nearly 3:1 OPO/SHG system when the frequency ratios slightly departs from the perfect 3:2:1 division ratio by a radio-frequency quantity $\delta<<\omega$, e.g. when $3 \omega \rightarrow 2 \omega-\delta, \omega+\delta$. The frequency difference beween the signal wave and the doubled idler is then $\left|2 \omega_{i}-\omega_{s}\right|=3 \delta$. When the doubled idler frequency does not match one of the cavity eigenmode frequencies, the 
operation of the OPO would be merely that of a conventional DRO. But if the OPO is tuned such that $3 \delta=F S R_{s} \approx$ $c / \Lambda, F S R_{s}$ being the signal free spectral range of the cavity, then the doubled idler will be enhanced to a point where it may lead to the creation of a new mode pair with frequencies $(2 \omega+2 \delta, \omega-2 \delta)$ and so on. The parametric gain bandwidth of OPOs extending usually over several $\mathrm{THz}$ or several tens of $\mathrm{THz}$ (in case of a wavelength non-critical phase matching), a multitude of self-phase locked mode pairs equally spaced by $3 \delta$ may potentially oscillate, provided that the pumping level is sufficiently high. Such a complex system opens the prospect of building a mode-locked, dual-band OPO frequency comb generator using cascaded second-order nonlinearities as the passive mode-locking mechanism. In the time domain, the output of such a system would consist of a train of short optical pulses with a repetion rate set by the $F S R_{s}$ intermode spacing, provided that the relative phase between adjacent mode pairs is preserved and group velocity dispersion is compensated. We note that a similar cw-DRO running near frequency degeneracy with thousands of mode pairs actively locked by an intracavity electro-optic phase modulator has been recently reported, with a striking passive output stability feature compared to a conventional quasi-degenerate singlemode pair DRO [38].

Acknowledgement 1 This work is partially supported by an INCO-Copernicus European network program (contract $n^{\circ}$ ERBIC 15CT 980814 ).

\section{VII.}

\section{APPENDIX I: LINEAR STABILITY ANALYSIS OF THE SPL-DRO}

With $A_{j}(t) \equiv A_{j}(t, 0)$, Eqs. (9-11) give rise to the mapping equations

$$
\begin{aligned}
A_{2}(t+\tau) & \left.=r_{2} e^{i \Delta_{2}}\left[A_{2}(t)+i A_{p}(t) A_{2}^{*}(t)+i \chi^{*} A_{1}^{2}(t)\right]\right] \\
A_{1}(t+\tau) & =r_{1} e^{i \Delta_{2}}\left[A_{1}(t)+i A_{p}(t) A_{1}^{*}(t)+i \chi A_{2}(t) A_{1}^{*}(t)\right] \\
A_{p}(t) & =A_{i n}
\end{aligned}
$$

the stationary solutions of which are deduced for large $r_{1,2}$ and small $\Delta_{1,2}$. (See Eqs.(19),(22)-(23)).

The linear stability analysis consists in assuming small deviations $\delta A_{1,2}(t)$ from the stationary solutions $\bar{A}_{1,2}$,

$$
\begin{aligned}
A_{1,2}(t) & =\bar{A}_{1,2}+\delta A_{1,2}(t) \\
\delta A_{1,2}(t) & =\sum_{\lambda} \delta A_{1,2}(\lambda) e^{\lambda t}
\end{aligned}
$$

where $\lambda$ may be complex. The stationary solutions $\bar{A}_{1,2}$ are stable only if the real part of any $\lambda$ is negative. At the instability threshold, therefore the system may undergo a Hopf bifurcation $\left(\lambda_{0}= \pm i \beta\right)$, so that the intensitiy oscillates with time at angular frequency $\beta$.

Eqs. (A1-A4) lead to a linearized system of four equations, the determinant of which satisfies

$$
D=\left|\begin{array}{llll}
\Lambda+\alpha_{1} & -i \chi_{1} \bar{A}_{2} & -i \chi_{1} \bar{A}_{1}^{*} & -i \chi_{1} A_{i n, 1} \\
i \chi_{1}^{*} \bar{A}_{2}^{*} & \Lambda+\alpha_{1}^{*} & i A_{i n, 1}^{*} & i \chi_{1}^{*} \bar{A}_{1} \\
-2 i \chi_{2}^{*} \bar{A}_{1} & -i A_{i n, 2} & \Lambda+\alpha_{2} & 0 \\
i A_{i n, 2}^{*} & 2 i \chi_{2} \bar{A}_{1} & 0 & \Lambda+\alpha_{2}^{*}
\end{array}\right|=0
$$

with the notations

$$
\Lambda=e^{\lambda \tau}-1
$$

and

$$
\begin{aligned}
\alpha_{1,2} & =\kappa_{1,2}-i \Delta_{1,2} \\
\chi_{1} & =e^{i \Delta_{1}} \chi r_{1}, \chi_{2}=e^{-i \Delta_{1}} \chi r_{2} \\
A_{i n, 1,2} & =r_{1,2} e^{i \Delta_{1,2}} A_{\text {in }}
\end{aligned}
$$


Then, the eigenvalues $\Lambda$ are solutions of the quartic characteristic equation

$$
\Lambda^{4}+\Phi_{3} \Lambda^{3}+\Phi_{2} \Lambda^{2}+\Phi_{1} \Lambda+\Phi_{0}=0
$$

where all the coefficients $\Phi_{i}$ are real

$$
\begin{aligned}
\Phi_{3}= & -2\left(\kappa_{1}+\kappa_{2}\right) \\
\Phi_{2}= & \left|\alpha_{1}\right|^{2}+\left|\alpha_{2}\right|^{2}+4 \kappa_{1} \kappa_{2}-2 r_{1} r_{2}\left|\bar{A}_{i n}\right|^{2} \cos \left(\Delta_{1}-\Delta_{2}\right)-\left|\chi r_{2} \bar{A}_{2}\right|^{2} \\
& +4 r_{1} r_{2}\left|\chi \bar{A}_{1}\right|^{2} \cos \left(\Delta_{1}+\Delta_{2}\right) \\
\Phi_{1}= & 2 r_{1} r_{2}\left|\bar{A}_{i n}\right|^{2}\left[\left(\Delta_{1}+\Delta_{2}\right) \sin \left(\Delta_{1}+\Delta_{2}\right)-\left(\kappa_{1+} \kappa_{2}\right) \cos \left(\Delta_{1}-\Delta_{2}\right)\right]+2\left(\kappa_{2}\left|\alpha_{1}\right|^{2}+\kappa_{1}\left|\alpha_{2}\right|^{2}\right) \\
& -2 \kappa_{2}\left|\chi r_{2} \bar{A}_{2}\right|^{2}+4\left|\chi r_{1} r_{2} \bar{A}_{1}\right|^{2}\left[\left(\kappa_{1}+\kappa_{2}\right) \cos \left(\Delta_{1}+\Delta_{2}\right)+\left(\Delta_{1+} \Delta_{2}\right) \sin \left(\Delta_{1}+\Delta_{2}\right)\right] \\
& -i r_{1} r_{2}|\chi|^{2} \cos \Delta_{2}\left(\bar{A}_{i n} \bar{A}_{2}^{*} \bar{A}_{1}^{*}-c . c .\right)+3 r_{1} r_{2}|\chi|^{2} \sin \Delta_{2}\left(\bar{A}_{i n} \bar{A}_{2}^{*} \bar{A}_{1}^{*}+c . c .\right) \\
\Phi_{0}= & \left|\alpha_{1}\right|^{2}\left|\alpha_{2}\right|^{2}-2 r_{1} r_{2}\left|\bar{A}_{i n}\right|^{2}\left[\left(\kappa_{1} \kappa_{2}+\Delta_{1} \Delta_{2}\right) \cos \left(\Delta_{1}-\Delta_{2}\right)+\left(\Delta_{1} \kappa_{2}-\Delta_{2} \kappa_{1}\right) \sin \left(\Delta_{1}-\Delta_{2}\right)\right] \\
& +4 r_{1} r_{2}\left|\chi \bar{A}_{1}\right|^{2}\left[\left(\kappa_{1} \kappa_{2}-\Delta_{1} \Delta_{2}\right) \cos \left(\Delta_{1}+\Delta_{2}\right)+\left(\Delta_{1} \kappa_{2}+\Delta_{2} \kappa_{1}\right) \sin \left(\Delta_{1}+\Delta_{2}\right)\right] \\
& -\left|\chi r_{1} \alpha_{2} \bar{A}_{2}\right|^{2}+\left(4\left|\chi r_{2} \bar{A}_{1}\right|^{2}-r_{2}^{2}\left|\bar{A}_{i n}\right|^{2}\right)\left(\left|\chi r_{1} \bar{A}_{1}\right|^{2}-r_{1}^{2}\left|\bar{A}_{i n}\right|^{2}\right)
\end{aligned}
$$

The roots for $\Lambda$ and consequently the eigenvalues $\lambda$ have been calculated for various detunings $\Delta_{1,2}$ as a function of the input amplitude $A_{i n}$ for the trivial solutions $\bar{A}_{1,2}=0$ and the non trivial solutions $\left(\bar{A}_{1,2}\right)_{ \pm}$deduced from Eqs.(16) and (20).

The trivial solution is verified to be stable for any input pump intensity below $I_{t h}$ (Eq.18).

On the other hand the solution $X_{-}$is found to be unstable with respect to any constant perturbation for any detuning and input pump intensity: Indeed, in this case, Eq.( A8) has a root $\Lambda=0$,i.e, $\lambda=0$, because the constant coefficient $\Phi_{0}$ is identically nil, when replacing $X_{-}$by its expression (16).

Differently, the upper branch displays two sets of complex conjugate eigenvalues, $\left(\sigma_{1,2} \pm i \beta_{1,2}\right)$ with negative real parts for a range of the input pump intensity, lying from $I_{0}$ to $I_{H}$ where the system undergoes a Hopf bifurcation $\left(\sigma_{1}=0\right)$. For $I_{p}>I_{H}$, the signal and idler intensities oscillate with time at angular frequency $\beta_{1}$. Therefore the upper branch is stable for $I_{0}<I_{p}<I_{H}$.

The Hopf bifurcation threshold intensity $I_{H}$ may be either smaller or larger than the threshold intensity $I_{t h}$, depending on detunings $\Delta_{1}$ and $\Delta_{2}$. Let us introduce the parameter $\mu_{H}$ that measures the departure between the threshold for lasing $I_{t h}$ and the Hopf bifurcation threshold as

$$
\mu_{H}=\left(I_{t h}-I_{H}\right) / I_{t h}
$$

The variation of $\mu_{H}$ is presented in Fig. 11 as a function $\Delta_{1}=\Delta$ either with $\Delta_{1}=\Delta_{2}$ in curve (a) or $\Delta_{1}=\frac{1}{2} \Delta_{2}$ in curve (b).

For $\Delta_{1}=\Delta_{2}$, the Hofp bifurcation $\left(\sigma_{1}=0, \sigma_{2}<0\right)$ occurs above threshold only for $\Delta \lesssim \kappa$. Therefore lasing stationary solutions can be reached from rest only for detunings smaller than the cavity loss coefficient. Otherwise, lasing at larger detunings can be reached, when increasing adiabatically the detunings from values smaller than $\kappa$. In the other case, $\Delta_{1}=\frac{1}{2} \Delta_{2}, \mu_{H}$ is positive for any $\Delta_{1} \lesssim 5 \kappa$. For higher detuning, $\sigma_{2}$ reaches zero at an input intensity much smaller than the value at which $\sigma_{1}=0$ crosses zero. This causes an abrupt decrease of $I_{H}$, so that $\mu_{H}$ suddenly becomes negative for $\Delta_{1} \geqslant 5 \kappa$, as shown in the curve (b) of Fig. 11.

Finally, the angular frequency $\beta_{H}$ at the Hopf bifurcation threshold, not reported here, is found to vary proportionally to the detuning. 


\section{Figure captions}

Fig.1: Schematic ring cavity model of SPL-OPOs. All intracavity losses are lumped into the output mirror transmissivities $t_{j}$.

Fig.2: Comparison of the meanfield and numerical stationary solutions of SPL-DRO, with $\kappa_{1,2}=\Delta_{1,2}=0.005$, as a function of the scaled pump input amplitude $\left(\alpha_{t h}=\sqrt{I_{t h}}\right)$. (a): meanfield upper branch (The dotted portion corresponds to unstable solutions), (a'): meanfield lower branch . (b): numerical solution for $|\chi|=0.05$. (c): numerical solutions for $|\chi|=0.2$.

Fig.3:Numerical SPL-DRO self locking ranges as a function of the detuning $\Delta_{1} / \kappa_{1}\left(=\Delta_{2} / \kappa_{2}\right)$, for $I_{\text {in }}=4 \kappa_{1} \kappa_{2}$, with $|\chi|=0.01$ in (a), 0.05 in (b), 0.1 in (c), 0.2 in (d) and 0.25 in (e). The dashed line is for $|\chi|=0$. The parameters are $\kappa_{1,2}=0.005$.

Fig.4: Time response of the SPL-DRO stationary state operating at $\left\{|\chi|=0.2, \bar{\Delta}_{\text {opt }}=4\right\}$, under step-wise linear detuning jumps $\bar{\Delta}_{\text {opt }}(1 \pm \beta$ ), with modulation index $\beta=0.109$ (curve a). Curve (b) gives the idler amplitude response and curve (c) the phase difference response. The re-capture range of the detuning perturbation is $\beta_{\max }=0.12$.

Fig.5: Contour plots of the stationary SPL-DRO signal (a), idler (b), and total (c) intensities and phase difference $\eta$ (d) in the $2 \mathrm{D}$ detunig plane, obtained from the numerical computation with $\kappa_{1,2}=0.005,|\chi|=S=0.2, I_{\text {in }}=4 \kappa_{1} \kappa_{2}$ . The intensities are scaled to $I_{1,2}=2 \kappa_{2,1}$. The pale blue domain are trivial solutions.

Fig.6: Numerically computed distribution of stationary signal/idler phase difference as defined in eq.(14d) versus the initial random phase for $\Delta_{1,2}=\kappa_{1,2}=0.005, \alpha_{i n} / \alpha_{t h}=2$ and $S=0.001$, for a SHG phase mismatch $\xi=\pi$ (i.e $\chi=0)$ in blank circles; $\xi=0$ in solid black circles and $\xi=3 \pi / 2$ in black triangles. Note that each phase data point is associated to the same stationary signal-idler intensities and the same phase sum $\mu$.

Fig.7: Time-dependent numerical solutions for $\kappa_{1}=0.005, \kappa_{2}=0.002, \Delta_{1,2}=0, \chi=0.2, I_{\text {in }}=1.96 \kappa_{1} \kappa_{2}$ (bottom frame) and $I_{i n}=4 \kappa_{1} \kappa_{2}$ (top frame).

Figs.8: Meanfield and numerical SPL-TRO stationary solutions for $\Delta_{p}=0, r_{p}=0.9$ and the same other parameters as for Fig.2. The top frame shows details of the hysteresis loop of the idler amplitude in the vicinity of the input pump threshold. The thin lines correspond to the numerical solutions, interrupted by the vertical thin dashed line. The thick lines correspond to the meanfield solutions. The thick dashed line in the bottom frame is the meanfield unstable branch of $\alpha_{p} / \alpha_{t h}\left(\alpha_{t h}=\sqrt{\kappa_{1} \kappa_{2}+\Delta_{1} \Delta_{2}}\right)$, and the thinner horizontal dashed line shows the conventional TRO pump clamping.

Fig.9: Subcriticality in the strongly pump-detuned conventional TRO $(S=0)$ and SPL-TRO $(S=0.2,0.6)$ for $r_{p}=0.9, \bar{\Delta}=1, \bar{\Delta}_{p}=2$ and same other parameters as in Fig.2. The thresholds located at the vertical arrows, are lower than the one given by eq.(27).

Fig.10: Contour plots of the numerical stationary SPL-TRO signal (a), idler (b), and total (c) intensities and phase difference $\eta(\mathrm{d})$ in the $2 \mathrm{D}$ detuning plane with $r_{p}=0.8, \Delta_{p}=0, \kappa_{1,2}=0.005, \chi=S=0.2, I_{\text {in }}=4 \kappa_{1} \kappa_{2}$. The intensities are scaled to $I_{1,2}=2 \kappa_{2,1}$. In the pale blue domain there is no lasing. For $r_{p}=0.9$, the self locking domain extends over the whole $2 \mathrm{D}$ frame.

Fig.11: Plots of the reduced Hopf bifurcation thresholds $\mu_{H}=\left(I_{t h}-I_{H}\right) / I_{t h}$ versus the normalised idler detuning $\Delta_{1} / \kappa_{1}$, for $\Delta_{2}=\Delta_{1}$ (solid line (a)); $\Delta_{2}=2 \Delta_{1}$ (solid line (b)). The cavity loss parameters used are $\kappa_{1,2}=\kappa=0.005$ and $S=0.2$.

[1] H.R. Telle, D. Meschede, and T.W. Hänsch, Opt. Lett. 15, 532 (1990).

[2] N.C. Wong, Opt. Lett. 17, 1155 (1992).

[3] S.A. Diddams et al, Phys. Rev. Lett. 84, 5102 (2000).

[4] T.W. Hänsch, Opt. Commun. 80, 71 (1990).

[5] T. Mukai, R. Wynands and T.W. Hänsch, Opt. Commun. 95, 71 (1993).

[6] Y. Kobayashi and K. Torizuka, Opt. Lett. 25, 856 (2000).

[7] A.E. Siegman, in Lasers (see chapt. 27,29), Univ. Science Books ed.(Sausalito, California ,1986).

[8] M. Hyodo, N. Onodera, and K.S. Abedin, Opt. Lett. 24, 303 (1999).

[9] O. Pfister et al, Opt. Lett. 21, 1387 (1997).

[10] D. Touahri et al, Opt. Commun. 133, 471 (1997).

[11] P.T. Nee and N.C. Wong, Opt. Lett. 23, 46 (1998).

[12] J.E. Bernard, B.G. Whitford, and L. Marmet, Opt. Lett. 24, 98 (1999). 
[13] R. Graham and H. Haken, Zeit. für Phys. 210, 276 (1968).

[14] D. Lee and N.C. Wong, Opt. Lett. 17, 13 (1992).

[15] S. Slyusarev, T. Ikegami and S. Oshima, Opt. Lett. 24, 1856 (1999).

[16] A. Douillet et al, to appear in IEEE Trans. Meas. \& Instr. (special volume on CPEM conf., Sydney, 2000).

[17] E. J. Mason and N.C. Wong, Opt. Lett. 23, 1733 (1998).

[18] D.-H. Lee et al, Opt. Express 5, 114 (1999).

[19] C.D. Nabors et al, Opt. Lett. 14, 1189 (1989).

[20] C.D. Nabors, S.T. Yang, T. Day, and R.L. Byers, J. Opt. Soc. Am. B7, 815 (1990).

[21] C. Fabre, E.J. Mason, and N.C. Wong, Opt. Commun. 170, 299 (1999).

[22] P.P. Bey and C.L. Tang, IEEE J. Quantum Electron. QE-8, 361 (1972).

[23] G.T. Moore, K. Koch, and E.C. Cheung, Opt. Commun. 113, 463 (1995); J. Opt. Soc. Am. B12, 2268 (1995).

[24] G.T. Moore and K. Koch, IEEE J. Quantum Electron. 29, 961 (1993).

[25] Y. Dikmelik and O. Aytür, IEEE J. Quantum Electron. 35, 897 (1999).

[26] A.G. White et al, Phys. Rev. A55, 4511 (1997).

[27] M.A.M. Marte, J. Opt. Soc. Am. B12, 2296 (1995).

[28] L. Zhang, Q. Zheng, and T. Kobayashi, J. Opt. Soc. Am. B14, 979 (1997).

[29] L.A. Lugiato, C. Oldano, C. Fabre, E. Giacobino and R.J. Horowicz, Il Nuovo Cimento 10D, 959 (1988).

[30] T. Debuisschert, A. Sizmann, E. Giacobino, and C. Fabre, J. Opt. Soc. Am. B10, 1668 (1993).

[31] L. Lugiato, in Progress in Optics, .E. Wolf ed.(North-Holland, Amsterdam, 1984), Vol. XXI, p.69.

[32] M. Le Berre, A.S. Patrascu, E Ressayre, A. Tallet and N.I. Zheleznykh, Chaos, Solitons, \& Fractals 4, 1389 (1994).

[33] M. Tlidi, M. Le Berre, E. Ressayre, A. Tallet, and L. Di Menza, Phys. Rev. A61, 043806 (2000).

[34] L.R. Brothers, D. Lee, and N.C. Wong, Opt. Lett. 19, 245 (1994).

[35] C. Ritchy, K.I. Petsas, E. Giacobino, C. Fabre and L. Lugiato, J. Opt. Soc. Am. B12, 456 (1995).

[36] F.G. Colville, M.J. Padgett, and M.H. Dunn, Appl. Phys. Lett. 64, 1490 (1994).

[37] G.-L. Oppo, M. Brambilla, and L.A. Lugiato, Phys. Rev. A49, 2028 (1994).

[38] S.A. Diddams, L.-S. Ma, J. Ye, and J.L. Hall, Opt. Lett. 24, 1747 (1999). 
This figure "fig1.jpg" is available in "jpg" format from: http://arxiv.org/ps/physics/0011034v1 
This figure "fig2.jpg" is available in "jpg" format from: http://arxiv.org/ps/physics/0011034v1 
This figure "fig3.jpg" is available in "jpg" format from: http://arxiv.org/ps/physics/0011034v1 
This figure "fig4.jpg" is available in "jpg" format from: http://arxiv.org/ps/physics/0011034v1 
This figure "fig5.jpg" is available in "jpg" format from: http://arxiv.org/ps/physics/0011034v1 
This figure "fig6.jpg" is available in "jpg" format from: http://arxiv.org/ps/physics/0011034v1 
This figure "fig7.jpg" is available in "jpg" format from: http://arxiv.org/ps/physics/0011034v1 
This figure "fig8.jpg" is available in "jpg" format from: http://arxiv.org/ps/physics/0011034v1 
This figure "fig9.jpg" is available in "jpg" format from: http://arxiv.org/ps/physics/0011034v1 
This figure "fig10.jpg" is available in "jpg" format from: http://arxiv.org/ps/physics/0011034v1 
This figure "fig11.jpg" is available in "jpg" format from: http://arxiv.org/ps/physics/0011034v1 